\title{
Exploring the LHC medium with direct photons
}

\author{
A. Marín ${ }^{\mathrm{a}}$ \\ for the ALICE Collaboration \\ GSI, Planckstrasse 1, 64291 Darmstadt, Germany
}

Received: 18 September 2008 / Revised: 16 January 2009 / Published online: 13 February 2009

(C) Springer-Verlag / Società Italiana di Fisica 2009

\begin{abstract}
Heavy-ion collisions will enter a new era with the start of the CERN Large Hadron Collider (LHC). A first short run with proton-proton collisions at the injection energy of $0.9 \mathrm{TeV}$ will be followed by a longer one with $p p$ collisions at $10 \mathrm{TeV}$. First $\mathrm{Pb}-\mathrm{Pb}$ collisions at $\sqrt{s_{N N}}=$ $5.5 \mathrm{TeV}$ will take place in 2009. Three experiments (ALICE, ATLAS, and CMS) will study both $p p$ and $\mathrm{Pb}-\mathrm{Pb}$ collisions. A selection of results showing the capabilities of the three experiments for the study of the LHC medium with direct photons is presented.
\end{abstract}

PACS 25.75.-q $\cdot 13.85 . \mathrm{Qk} \cdot 25.75 . \mathrm{Cj}$

\section{Introduction}

With the startup of the CERN LHC heavy-ion collisions will enter a new era. With a center of mass energy of $5.5 \mathrm{TeV}$, about a factor 30 larger than the available energy at RHIC, matter will be produced in a new domain of high energy density ( 15 to $60 \mathrm{GeV} / \mathrm{fm}^{3}$ ) and high initial temperature (factor 3 to 5 higher than the critical Temperature, $T_{c}$ ). At high temperature and high energy density QCD calculations $[1,2]$ predict a phase transition from hadron gas to quark gluon plasma (QGP) and chiral symmetry restoration. The expected time the system will expend in the QGP phase is of the order of $10 \mathrm{fm} / \mathrm{c}$ and it is expected to extend over a large volume. The cross sections of hard probes are increasing by large factors at LHC energies compared to RHIC or SPS [3]. Among the different observables photons and dileptons are particularly interesting because they would directly probe the high temperature and high density phase of these collisions. The $p p$ runs will provide a test of $\mathrm{pQCD}$ as well as important reference data for heavy-ion runs. With the first heavy-ion run expected in 2009, the global event characteristics and bulk properties will be established, and hard probes measurements could be started.

a e-mail: a.marin@gsi.de

\section{Direct photon phenomenology at the LHC}

There are different production mechanisms of photons in heavy-ion collisions. Direct photons are produced at leading order (LO) in quark gluon Compton scattering $(\mathrm{qg} \rightarrow \mathrm{q} \gamma)$ and quark anti-quark annihilation ( $\mathrm{q} \bar{q} \rightarrow \mathrm{g} \gamma)$. The next-to-leading order (NLO) process is dominated by bremsstrahlung and fragmentation photons ( $\mathrm{qg} \rightarrow \mathrm{qg} \gamma$ ). In addition, there are photons from the jet re-interaction in the medium and thermal photons from QGP and from the hot hadronic stage following the QGP. Direct photons have to be isolated from the main source of background photons, i.e. the decay of hadrons (mainly $\pi^{0}$ 's and $\eta$ 's) after the QGP phase.

The different contributions to the direct photon spectrum for LHC energies as calculated by [4] is presented in Fig. 2.1. Other predictions are also available [5-8]. At RHIC $\gamma$ 's from the parton scattering at $\mathrm{LO}$ were the dominant contribution for $p_{T}>5 \mathrm{GeV} / \mathrm{c}$ while at LHC jet-photon conversion in the plasma dominates between 8 and $14 \mathrm{GeV} / \mathrm{c}$. Only for $p_{T}>20 \mathrm{GeV} / \mathrm{c}$ the hard NN scattering dominates. Although photons are more abundantly produced at LHC compared to RHIC (about a factor 10 larger), the ratio of direct photons to $\pi^{0}$ is smaller at LHC. The ratio is $\sim 10 \%$ for a $p_{T}$ of $20 \mathrm{GeV} / \mathrm{c}$ [9], thus a very good PID to distinguish between direct and decay photons would be essential.

Recently it has been proposed [10] that in order to distinguish between the different photon sources one can study their azimuthal anisotropy as function of $p_{T}$ in conjunction with $R_{A A}$. A calculation for semicentral $\mathrm{Pb}-\mathrm{Pb}$ collisions at LHC energies [11] predicts $v_{2}=0$ for initial production, positive for jet fragmentation and negative for jet photon conversion processes. Therefore, a negative $v_{2}$ together with $R_{A A}>1$ will be an unambiguous signature of medium produced photons.

The high $p_{T}$ photon measurement opens the possibility of studying the jet fragmentation function (FF) and its modification (redistribution of the parton energy inside the 


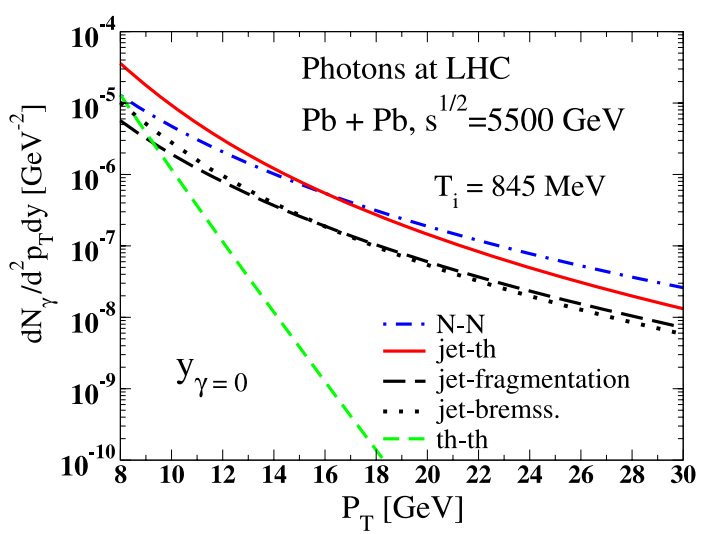

Fig. 2.1 Contributing sources of high- $p_{T}$ photons at midrapidity in central $\mathrm{Pb}-\mathrm{Pb}$ collisions at $\mathrm{LHC}$ from [4]

hadron jet) due to the medium effects in heavy-ion collisions by using the $\gamma$-jet channel. As the photon is not affected by final state interactions, its transverse energy $\left(E_{T}\right)$ gives the energy of the jet before modification in the medium. This measurement would be complementary to jet reconstruction in the energy range from 20 to $80 \mathrm{GeV}$ [12], or above $75 \mathrm{GeV}[13,14]$.

\section{ALICE, ATLAS and CMS as photon and dilepton detectors at the $\mathrm{LHC}$}

Three experiments will participate in the heavy-ion program at the CERN LHC. ALICE is the experiment devoted to the study of heavy-ion collisions $[15,16]$, and it will also participate in the $p p$ running with an extensive physics program. ATLAS [17] and CMS [18] are experiments dedicated to study $p p$ collisions although by now they also have a rich heavy-ion program $[19,20]$.

The ALICE detector (Fig. 3.1 top) $[15,16]$ consists of central barrel detectors, a forward muon spectrometer and forward multiplicity and centrality detectors. The central barrel inside a large solenoid magnet with the field up to $0.5 \mathrm{~T}$ covers almost 2 units of pseudo-rapidity $(|\eta|<0.9)$. The charged particle tracking detectors are the ITS (Inner Tracking System) with three different silicon technologies (pixels, drift and strips), the TPC (Time Projection Chamber) main tracking system for charged particles, and the TRD (Transition Radiation Detector). All three tracking detectors are also used for particle identification (PID) by measuring ionization losses or transition radiation. The TOF (Time Of Flight) detector is used for PID in the intermediate momentum range $(0.2$ to $2.5 \mathrm{GeV} / \mathrm{c})$. Three smaller single arm detectors complete the central barrel, the HMPID (High Momentum Particle Identification) detector that will extend the PID up to momenta of $4-5 \mathrm{GeV} / \mathrm{c}$, the high resolution high granularity electromagnetic calorimeter PHOS (PHOton Spectrometer) to measure photons and neutral mesons
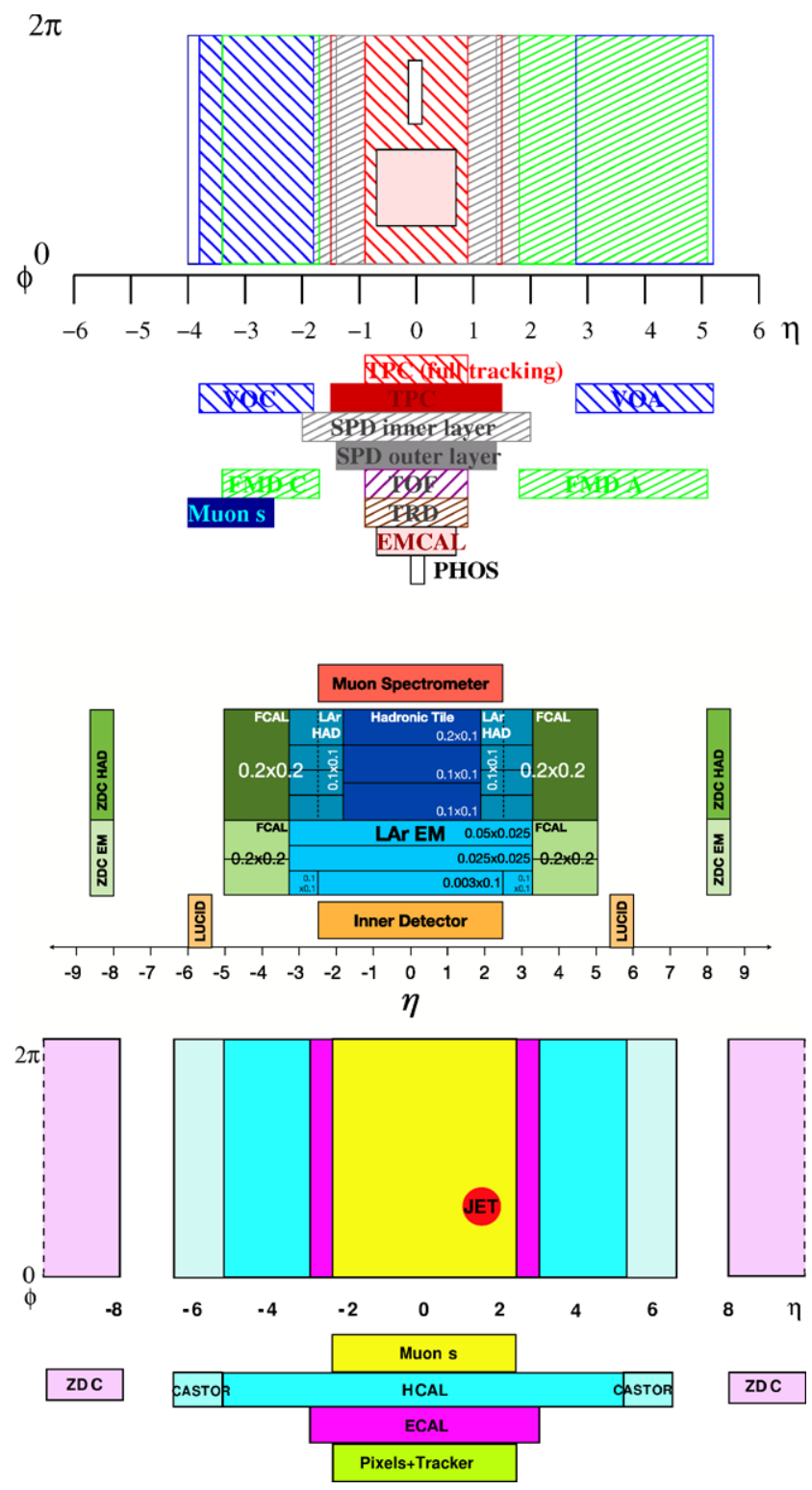

Fig. 3.1 ALICE (top), ATLAS (middle), and CMS (bottom) pseudorapidity and azimuthal coverage

and the EMCAL (ElectroMagnetic CALorimeter) to improve the ALICE capabilities in the measurement of high energy jets and direct photons. At forward rapidities, ALICE triggers and detects muons using the muon spectrometer with tracking and trigger chambers in a $3 \mathrm{Tm}$ magnetic field. ALICE has an excellent PID: $\pi / \mathrm{K} / p$ identification up to $50 \mathrm{GeV} / \mathrm{c}$ and $\gamma, e$, and $\mu$ up to $100 \mathrm{GeV} / \mathrm{c}$. The ALICE specific photon detectors are PHOS and the EMCAL. In addition, photons that convert in the detector material $\left(\gamma \mathrm{Z} \rightarrow \mathrm{e}^{-} \mathrm{e}^{+} \mathrm{Z}\right)$ can be detected by measuring the $\mathrm{e}^{+}$and $\mathrm{e}^{-}$in the central barrel. This is a clean photon identification method, providing directional information used to reject non vertex background. The momentum resolution at low $p_{T}$ using this method is better than electromagnetic 
Table 3.1 Compilation of the photon detectors in the three LHC experiments with a brief description of their characteristics

\begin{tabular}{|c|c|c|c|c|c|c|c|}
\hline \multirow{2}{*}{$\begin{array}{l}\text { Exp } \\
\text { Name }\end{array}$} & \multicolumn{2}{|l|}{ ATLAS } & \multicolumn{2}{|l|}{ CMS } & \multicolumn{3}{|l|}{ ALICE } \\
\hline & LAr Barrel & LArEndCap & ECAL(EB) & ECAL(EE) & PHOS & EMCAL & Barrel \\
\hline Coverage & $\begin{array}{l}0<|\eta|<1.4 \\
2 \pi\end{array}$ & $\begin{array}{l}1.4<|\eta|<3.2 \\
2 \pi\end{array}$ & $\begin{array}{l}0<|\eta|<1.5 \\
2 \pi\end{array}$ & $\begin{array}{l}1.5<|\eta|<3 \\
2 \pi\end{array}$ & $\begin{array}{l}0<|\eta|<0.12 \\
0.6 \pi\end{array}$ & $\begin{array}{l}0<|\eta|<0.7 \\
0.6 \pi\end{array}$ & $\begin{array}{l}(0<|\eta|<0.9 \\
2 \pi) \cdot 7 X / X_{0}\end{array}$ \\
\hline $\begin{array}{l}\text { Granularity } \\
\Delta \eta \times \Delta \phi\end{array}$ & $\begin{array}{l}0.003 \times 0.100 \\
0.025 \times 0.025 \\
0.025 \times 0.025\end{array}$ & $\begin{array}{l}0.025 \times 0.100 \\
0.025 \times 0.025 \\
0.025 \times 0.025\end{array}$ & $0.017 \times 0.017$ & $\begin{array}{l}0.017 \times 0.017 \\
\text { to } \\
0.05 \times 0.05\end{array}$ & $0.004 \times 0.004$ & $0.014 \times 0.014$ & $\begin{array}{l}3 \times 10^{-4} \times 2 \times 10^{-4} \\
\text { resolution }\end{array}$ \\
\hline Resolution & $\begin{array}{l}10 \% / \sqrt{E} \bigoplus \\
0.5 \%\end{array}$ & $\begin{array}{l}10 \% / \sqrt{E} \oplus \\
0.5 \%\end{array}$ & $\begin{array}{l}2.7 \% / \sqrt{E} \oplus \\
0.55 \%\end{array}$ & $\begin{array}{l}5.7 \% / \sqrt{E} \oplus \\
0.55 \%\end{array}$ & $\begin{array}{l}1.3 \% / \sqrt{E} \oplus \\
1.1 \%\end{array}$ & $\begin{array}{l}7 \% / \sqrt{E} \oplus \\
1.5 \%\end{array}$ & $\begin{array}{l}2 \% \text { low pt } \\
5 \% \text { high pt }\end{array}$ \\
\hline
\end{tabular}

calorimeters. The possibility of using a L1 TRD trigger [21] to make use of this method at high $p_{T}$ is under investigation.

The ATLAS detector (Fig. 3.1 middle) [17, 19] has hermetic azimuthal coverage over a wide range in pseudorapidity. The unique feature of the ATLAS detector is its calorimetry, composed of several independent longitudinal sampling layers of electromagnetic and hadronic calorimetry with full azimuthal and $|\eta|<5$ units coverage. Specifically, the Liquid Argon (LAr) electromagnetic calorimeter provides excellent energy and position information on electrons and photons. The inner tracking system within a $2 \mathrm{~T}$ solenoidal field is equipped with silicon pixels, silicon strips and straw-tube transition-radiation tracker for reconstructing charged tracks within $|\eta|<2.5$. A muon spectrometer $(|\eta|<3)$ is located within a toroidal field outside of the hadronic calorimeters.

The CMS detector (Fig. 3.1 bottom) $[18,20]$ is also a hermetic detector with a large acceptance both for tracker and calorimetry. The CMS detector is a $22 \mathrm{~m}$ (length) $\times$ $15 \mathrm{~m}$ (diameter) detector featuring a $4 \mathrm{~T}$ solenoid surrounding central silicon pixel and micro-strip tracking detectors $(|\eta|<2.4)$ and electromagnetic $(|\eta|<3)$ and hadronic $(|\eta|<5)$ calorimeters. Muon detectors $(|\eta|<2.4)$ are embedded in the flux return iron yoke of the magnet. CMS detects leptons and both charged and neutral hadrons.

A compilation of the different photon detectors with their main characteristics in the three LHC experiments is presented in Table 3.1. PHOS and LAr (Barrel) have the best granularity.

\section{Prompt photons and photon-tagged jets}

To demonstrate the direct photon reconstruction capabilities ALICE has generated events using PYTHIA [22] triggered by prompt photons ( $\gamma$-jet events) or $\pi^{0}$ (jet-jet events). To simulate $\mathrm{Pb}-\mathrm{Pb}$ events, the $p p$ events were merged with heavy-ion events generated by HIJING [23, 24]. Prompt

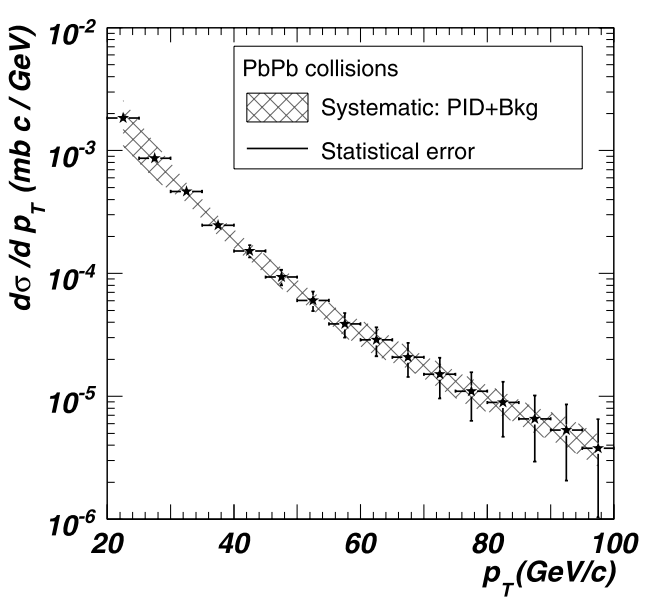

Fig. 4.1 Simulated prompt photon spectrum expected to be measured in ALICE during a LHC running year for $\mathrm{Pb}-\mathrm{Pb}$ collisions with statistical (bars) and systematic (shaded band) errors

photons are selected in PHOS by applying cuts on the shape of the shower developed on the detector and isolation cuts $[25,26]$.

The spectrum of direct photons detected in PHOS for $\mathrm{Pb}$ $\mathrm{Pb}$ collisions $[25,26]$ is shown in Fig. 4.1. An isolation cut of radius $R=\sqrt{\Delta \eta^{2}+\Delta \phi^{2}}=0.2$ and a $p_{T}$ threshold of $2 \mathrm{GeV} / \mathrm{c}$ is used. The background rejection with the applied cuts is $1 / 14$ for an efficiency of $50 \%$. A sample of $2000 \gamma$ with $p_{T}>20 \mathrm{GeV} / \mathrm{c}$ is expected during one LHC running year.

The spectrum of prompt photons detected in 2 PHOS modules for $p p$ collisions is presented in Fig. 4.2. An isolation cut with $R=0.3$ and $\sum p_{T}<2 \mathrm{GeV} / \mathrm{c}$ has been used. A background rejection of $1 / 170$ is obtained for an efficiency of $69 \%$. A sample of $3000 \gamma$ with $p_{T}>20 \mathrm{GeV} / \mathrm{c}$ is expected during one LHC running year $\left(10 \mathrm{pb}^{-1}\right)$ [27].

As direct photons are not perturbed by the medium the fragmentation function of the recoiled jet can be measured using $E_{\gamma}$ as the jet energy. The ratio of the measured fragmentation functions for the two collision systems shows that for $E>20 \mathrm{GeV}$ variations of $\mathrm{R}_{F F}$ larger than $5 \%$ in 


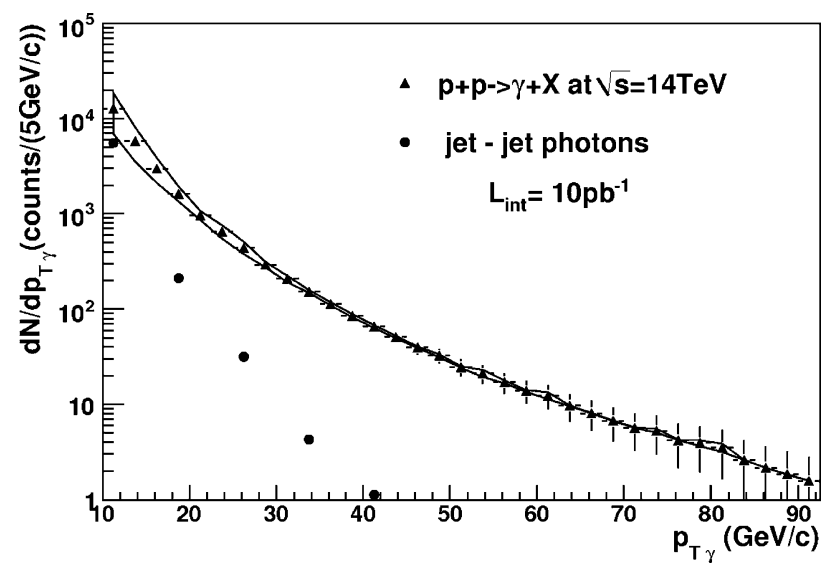

Fig. 4.2 Isolated photon spectrum that can be measured in ALICE with 2 PHOS modules in $p p$ collisions at $14 \mathrm{TeV}$. The error bars are statistical errors. Systematic errors are given by the area around the data points

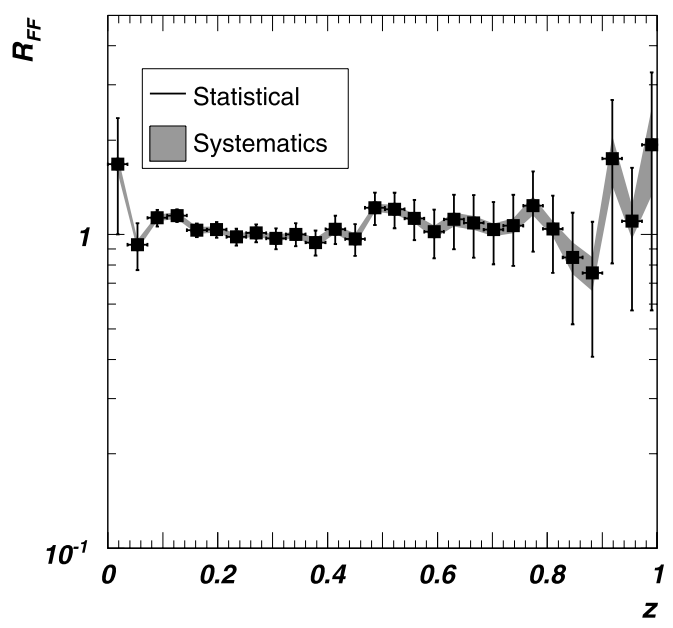

Fig. 4.3 Ratio of the fragmentation functions of $\gamma$-tagged jets with energy larger than $20 \mathrm{GeV}$ for $\mathrm{Pb}-\mathrm{Pb}$ collisions scaled to $p p$ collisions detected in the central tracking system and EMCal. The shaded region represents the systematic error due to the contamination from jet-jet events

the range $0.1<z=p_{T} / E_{j e t}<0.5$ can be measured, see Fig. 4.3.

The expected $p_{T}$ reach of the prompt photon spectrum will be larger in ATLAS and in CMS compared to ALICE due to their larger acceptance.

Due to the fact that the ATLAS detector has very good granularity in the first layer, isolated photons give a very clear signal even in a heavy-ion background simulated using HIJING. The set of cuts based on shape of the signal and isolation cuts used for this study give an efficiency of about $\sim 60 \%$ and a rejection factor of $\sim 10$ assuming $R_{A A}^{h} / R_{A A}^{\gamma}=1$. The direct photon spectrum resulting from combining shape and isolation cuts is presented in Fig. 4.4 [14, 28]. A sample of about 100K photons with

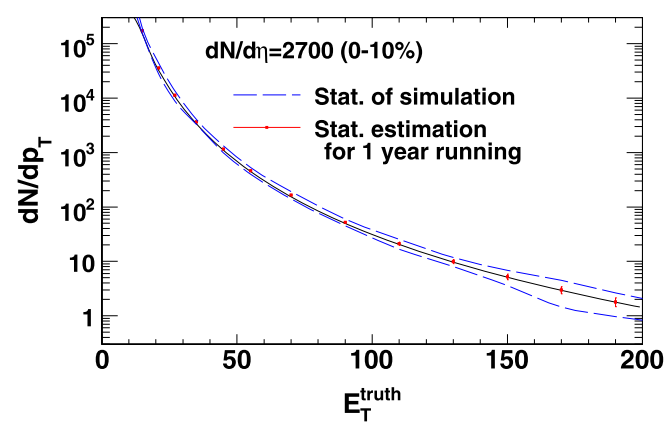

Fig. 4.4 Isolated photon spectrum as expected to be measured by the ATLAS detector in one LHC year running [28]

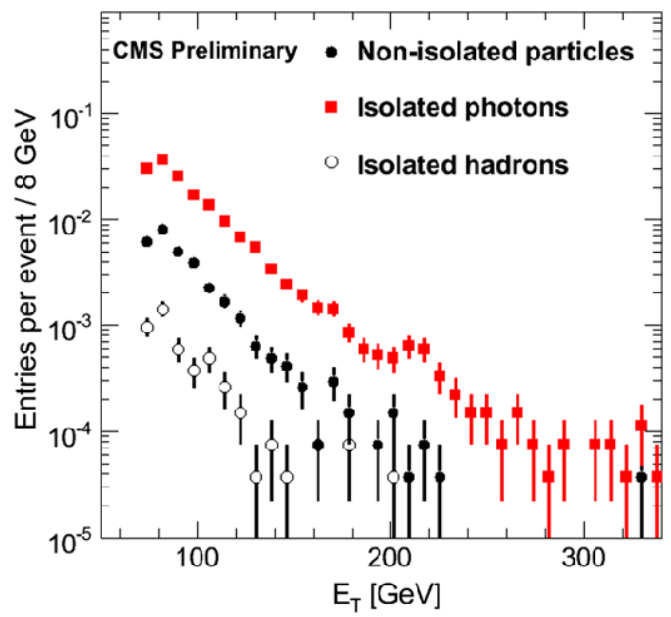

Fig. 4.5 Isolated photon spectrum as expected to be measured by $\mathrm{CMS}$ in one year of $\mathrm{Pb}-\mathrm{Pb}$ collisions [13]

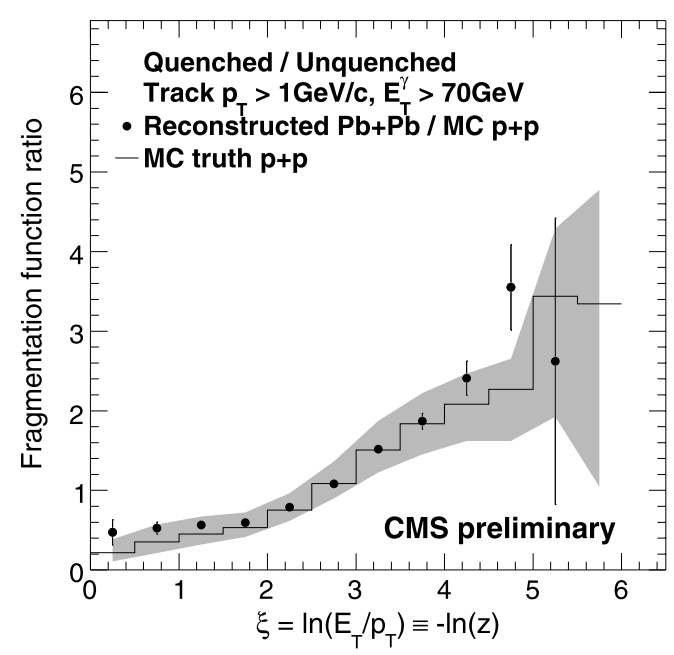

Fig. 4.6 Ratio of reconstructed (symbols) and MC truth (line) quenched fragmentation function over unquenched MC truth in CMS. The estimated systematic error is given by the shared band [13]

$E>30 \mathrm{GeV}$ and of about $10 \mathrm{~K}$ with $E>70 \mathrm{GeV}$ are expected for an integrated luminosity of $0.5 \mathrm{nb}^{-1}$. 
CMS simulation studies are performed using PYQUEN [29] and PYTHIA to generate the prompt photons with and without jet quenching respectively, and HYDJET [30] to model the underlying heavy-ion event. The working point of the photon detection is set to $60 \%$ signal efficiency, leading to a background rejection of about $96.5 \%$. The photon isolation and shape cuts improved S/B by a factor 15 . A detailed description of the photon detection method can be found in [13]. The photon spectrum for an integrated luminosity of $0.5 \mathrm{nb}^{-1}$ is shown in Fig. 4.5.

Isolated photons are associated with the back-to-back jet in order to reconstruct the fragmentation function. The ratio of the reconstructed fragmentation functions in quenched $\mathrm{Pb}-\mathrm{Pb}$ and in $p p$ is shown in Fig. 4.6. CMS obtains high significance for $\xi$ between 0.2 and 5 for $E_{\gamma}$ larger than $70 \mathrm{GeV}$ (Fig. 4.6).

In addition to the $\gamma$-tagged jet measurement, jets can also be tagged with a virtual photon or a $Z^{0}$. This method is free from the bias of the isolation cut that is applied in $\gamma$-tagged jets. A detailed study for the CMS detector is presented in $[20,31]$.

\section{Low mass dileptons}

The measurement of direct photons at low $p_{T}$ is usually performed on a statistical basis, namely subtracting the decay photons from all detected photons [32]. The difficulty of this method comes from the large background due to the decay photons. To overcome the background problem it has been proposed to consider instead the emission of virtual photons (lepton pairs). This method was used at CERN ISR to set a limit on direct photon production [33]. The invariant mass distribution of Dalitz decay pairs as well as of virtual photons is given by the Knoll-Wada formula [34].

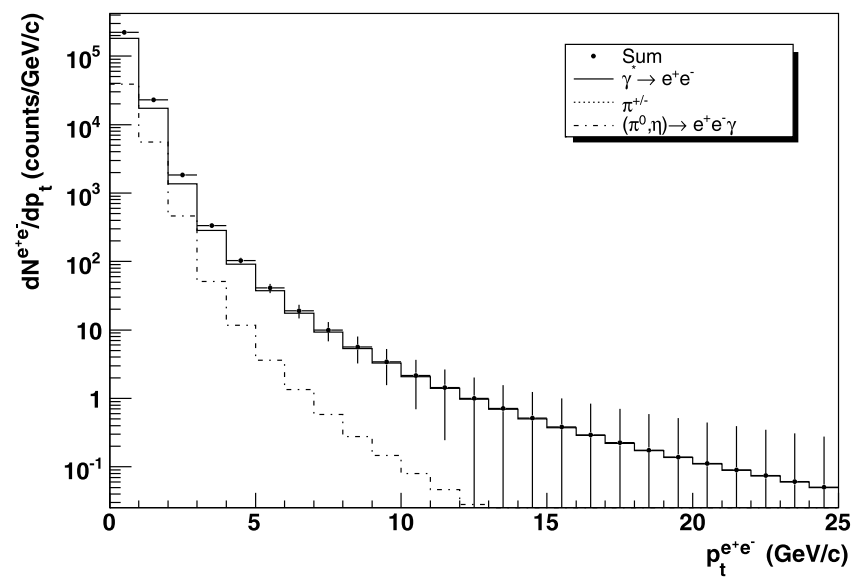

Beyond the mass of the $\pi^{0}$, only the $\eta$ mesons contributes. As most of the $\gamma$ 's come from $\pi^{0}$ decays, the signal to background ratio for direct photon signal improves considerably for $m_{e e}>m_{\pi^{0}}$. This method also benefits from the excellent mass resolution at low invariant mass and from the low conversion probability in the case of ALICE $(\sim 7 \%)$. For ATLAS and for CMS, where the conversion probability is higher ( $\sim 20 \%, \sim 30 \%)$, it would be more difficult to apply this method.

This method has also been used recently by PHENIX to measure direct photons in $p p$ and $\mathrm{Au}+\mathrm{Au}$ [35]. While the yield is consistent with NLO pQCD calculations in $p p$ collisions, in $\mathrm{Au}-\mathrm{Au}$ collisions the data are larger than calculations for $p_{T}<3.5 \mathrm{GeV} / \mathrm{c}$.

Theoretically, the rate of production of a dilepton pair in a finite mass range is given by [36]

$\frac{d \sigma^{e^{+} e^{-}}}{d p_{T} d y}=C_{e^{+} e^{-}} \alpha \frac{d \sigma^{\gamma}}{d p_{T} d y}$

where $C_{e^{+} e^{-}} \sim 0.3$ for $0.2 \mathrm{GeV} / \mathrm{c}^{2}<M_{e^{+} e^{-}}<0.6 \mathrm{GeV} / \mathrm{c}^{2}$ valid in the range $2 \mathrm{GeV} / c<p_{T}<100 \mathrm{GeV} / \mathrm{c}$. The expected yield of virtual photons calculated taking into account (1) and the pQCD rate for a LHC running year is shown in Fig. 5.1. Thermal photons are supposed to be the dominating contribution below $p_{T} \sim 5 \mathrm{GeV} / \mathrm{c}$ [5-7], therefore the dilepton yield is probably underestimated. Points for $p_{T}$ below $2 \mathrm{GeV} / \mathrm{c}$ should be taken with some caution, as this method assumes that the mass of the dilepton pair is negligible compared to the $p_{T}$ and the validity of (1). The electron pairs are detected and identified by the ALICE central tracking system (TPC and TRD).

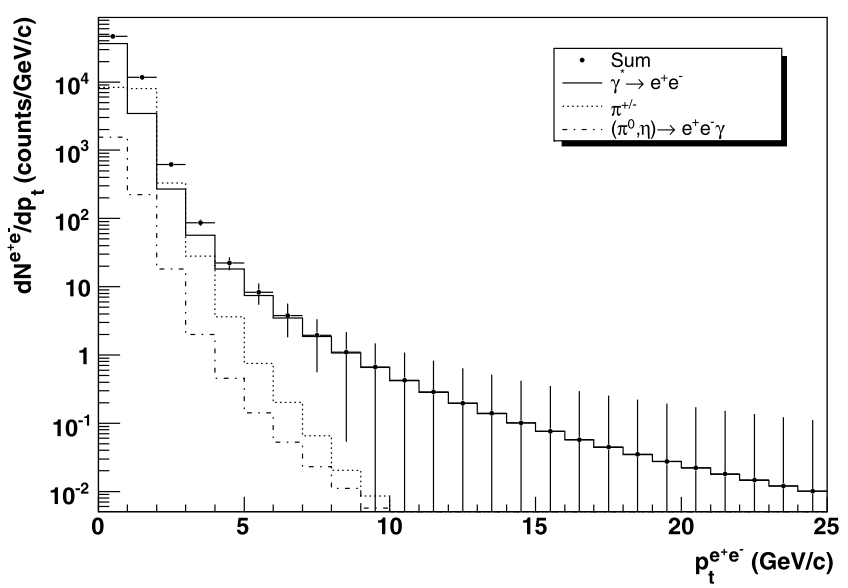

Fig. 5.1 Virtual photon spectrum reconstructed from the measurement of electron pairs with mass on the range 0.2 to $0.6 \mathrm{GeV} / \mathrm{c}^{2}$ in $\mathrm{pp}$ at $\sqrt{s}=5.5 \mathrm{TeV}($ left $)$ and in central $\mathrm{Pb}-\mathrm{Pb}$ collisions (right) at $\sqrt{s_{N N}}=5.5 \mathrm{TeV}$ 


\section{Conclusions}

The LHC machine and the experiments are finally a reality. We have shown that the three experiments are equipped with very good photon and dilepton detectors that will allow the measurement of electromagnetic probes at LHC energies from low to very high $p_{T}$. The low mass dilepton measurement will extend the direct photon spectrum to lower $p_{T}$. The jet fragmentation function and its modification due to the medium effects in heavy-ion collisions will be study using $\gamma$ tagged jets. The ALICE, ATLAS and CMS experiments will provide independent and complementary results, that will be essential to understand this new system.

Acknowledgements I would like to express my gratitude to the organizers of the 3rd International Conference on Hard and Electromagnetic Probes in High Energy Nuclear Collisions and the ALICE management for the invitation to speak in A Toxa. I would like also to thank the ALICE, the ATLAS and the CMS Collaborations for providing the material for this talk and for useful discussions. The author wishes to acknowledge the financial support received from GSI and from the German BMBF.

\section{References}

1. F. Karsch, hep-lat/0106019

2. M. Cheng et al., Phys. Rev. D 77, 014511 (2008)

3. C. Fabjan et al., in Proceedings of Quark Matter Conference 2008

4. S. Turbide et al., Phys. Rev. C 72, 014906 (2005)

5. R.J. Fries et al., Phys. Rev. Lett. 90, 132301 (2003)

6. S. Turbide et al., Phys. Rev. C 69, 014903 (2004)

7. F. Arleo et al., nucl-th/0707.2357v2

8. N. Armesto et al., J. Phys. G, Nucl. Part. Phys. 35, 054001 (2008)

9. F. Arleo et al., LHC CERN Yellow Report 2004-009. hep-ph/0311131

10. S. Turbide, et al., Phys. Rev. Lett. 96, 032303 (2006)
11. W. Liu, R.J. Fries, nucl-th/0801.0453

12. C. Klein-Boesing et al. (ALICE Collaboration), these proceedings

13. C. Loizides et al. (CMS Collaboration), in Proceedings of Quark Matter Conference 2008. arXiv0804.3679v1 [nucl-ex]

14. N. Grau et al. (ATLAS Collaboration), arXiv:0805.4656v1 [nuclex]

15. ALICE Collaboration et al., J. Phys. G, Nucl. Part. Phys. 30, 1517 (2004)

16. ALICE Collaboration et al., J. Phys. G, Nucl. Part. Phys. 32, 1295 (2006)

17. ATLAS Collaboration, ATLAS Technical Design Report, vol. 1 CERN/LHCC 1999-14, vol. 2 CERN/LHCC 1999-15

18. CMS Collaboration et al., The CMS Physics Technical Design Report, vol. 1, CERN/LHCC 2006-001

19. ATLAS Collaboration, Letter of Intent, Report CERN/LHCC/2004-009 Design Report, Addendum 1 CERN/LHCC 2007-009

20. D.G. d'Enterria et al. (CMS Collaboration), J. Phys. G, Nucl. Part. Phys. 34, 2307 (2007)

21. ALICE Collaboration et al., TRD Technical Design Report, CERN/LHCC 2001-021

22. T. Sjostrand et al., Comput. Phys. Commun. 135, 238 (2001)

23. X.N. Wang, N. Gyulassy, Phys. Rev. D 44, 3501 (1991)

24. M. Gyulassy, X.N. Wang, Comput. Phys. Commun. 83, 307 (1994)

25. G. Conesa et al., Nucl. Instrum. Methods A 580, 1446 (2007)

26. G. Conesa et al., Nucl. Instrum. Methods A 585, 28 (2008)

27. Y. Mao et al. (ALICE Collaboration), in Proceedings of Quark Matter Conference 2008. ALICE-INT, 021 (2007)

28. B.A. Cole et al. (ATLAS Collaboration), these proceedings

29. I.P. Lokhtin, A.M. Snigirev, Eur. Phys J. C 45, 211 (2006) [PYQUEN v1.2 is used]

30. I.P. Lokhtin, A.M. Snigirev, arXiv:hep-ph/0312204 [HYDJET v1.2 is used]

31. C.J. Kunde et al., these proceedings

32. D. Peressounko et al. (ALICE Collaboration), these proceedings

33. J.H. Cobb et al., Phys. Lett. B 78, 519 (1978)

34. N.M. Knoll, W. Wada, Phys. Rev. 98, 1355 (1955)

35. A. Adare et al. (PHENIX Collaboration), arXiv:0804.4168v1 [nucl-ex]

36. P. Aurenche et al., Phys. Lett. B 209, 375 (1988) 